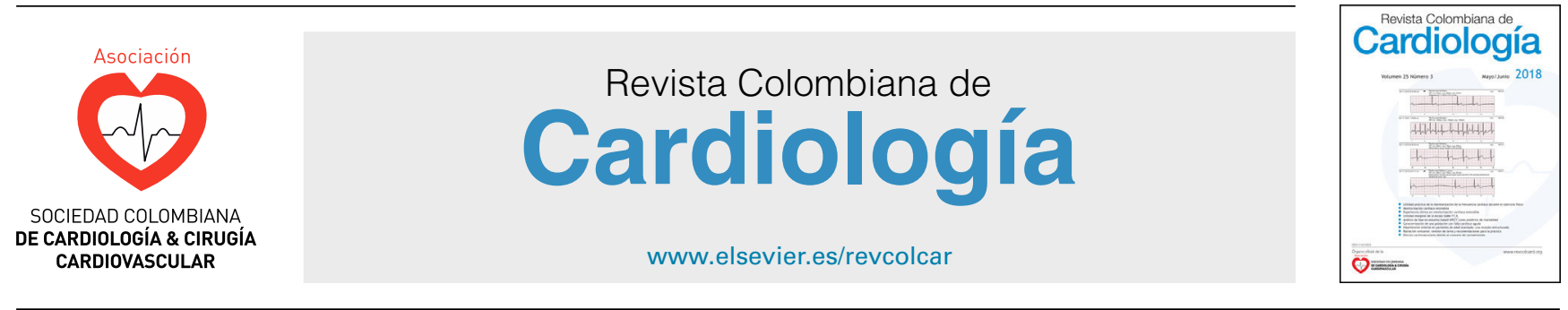

CARDIOLOGÍA DEL ADULTO - ARTÍCULO ORIGINAL

\title{
Validación para Colombia del cuestionario para la "Medición de la capacidad funcional en pacientes con falla cardíaca"'
}

\author{
Eliana M. Sánchez-Ropero ${ }^{a}$, Claudia Y. Vera-Giraldo ${ }^{a}$, Claudia M. Navas-Ríos ${ }^{b}$, \\ Sergio D. Ortiz-Rangel ${ }^{\mathrm{b}}$, Camila Rodríguez-Guevara ${ }^{\mathrm{a}, \mathrm{b}}$, Diana M. Vargas-Montoya ${ }^{\mathrm{b}}$, \\ Daniel Camilo Aguirre-Acevedo ${ }^{\mathrm{a}, \mathrm{c}}$ y Luz Helena Lugo-Agudelo ${ }^{\mathrm{a}, \mathrm{b}, *}$
}

\footnotetext{
a Grupo de Rehabilitación en Salud. Universidad de Antioquia, Medellín, Colombia

${ }^{\mathrm{b}}$ Medicina Física y Rehabilitación. Clínica Las Américas, Medellín, Colombia

c Grupo de Epidemiología Clínica. Universidad de Antioquia, Medellín, Colombia
}

Recibido el 8 de noviembre de 2017; aceptado el 6 de abril de 2018

Disponible en Internet el 11 de julio de 2018

\section{PALABRAS CLAVE \\ Falla cardiaca; \\ Estado funcional; \\ Validez; \\ Confiabilidad; \\ Índice de estado de \\ actividad de Duke}

\begin{abstract}
Resumen
Objetivos: Hacer la validación para Colombia del cuestionario "Índice de estado actividad de Duke en pacientes con falla cardíaca'.

Métodos: Se hizo la traducción y adaptación cultural del Índice de estado actividad de Duke (DASI) al español. Se incluyeron 135 pacientes, con quienes se hizo la validación de contenido con un análisis factorial confirmatorio. Se evaluó la validez de constructo convergente y divergente y la validez de criterio comparando grupos de la New York Heart Asociation (NYHA) y con las correlaciones con el cuestionario de calidad de vida "Minnesota Living with Heart Failure Questionnaire', la escala genérica de calidad de vida SF-36 y un cuestionario de evaluación de la discapacidad de la Organización Mundial de la Salud (WHO-DAS II). Se evaluó la consistencia interna y la fiabilidad interobservador e intraobservador, así como la sensibilidad al cambio al mes, tres y seis meses.

Resultados: En la validez de contenido la escala se ajusta a dos dominios; en la validez convergente y divergente se verificó con correlaciones significativas del DASI con las escalas con las que se comparó. La validez de criterio del DASI con la clasificación NYHA mostró relación adecuada, aunque no es estadísticamente significativa. En la consistencia interna el coeficiente de alfa de Cronbach fue de 0,69 y los coeficientes de correlación intraclase para la fiabilidad interevaluador e intraevaluador fueron 0,81 y 0,90 respectivamente.

Conclusiones: Esta versión en Español del Índice de estado actividad de Duke es válida y confiable para pacientes con falla cardíaca en Colombia.

(C) 2018 Sociedad Colombiana de Cardiología y Cirugía Cardiovascular. Publicado por Elsevier España, S.L.U. Este es un artículo Open Access bajo la licencia CC BY-NC-ND (http:// creativecommons.org/licenses/by-nc-nd/4.0/).
\end{abstract}

\footnotetext{
* Autor para correspondencia.

Correos electrónicos: gruporehabilitacionsalud@udea.edu.co,
} luzh.lugo@gmail.com (L.H. Lugo-Agudelo). https://doi.org/10.1016/j.rccar.2018.04.004 0120-5633/৫ 2018 Sociedad Colombiana de Cardiología y Cirugía Cardiovascular. Publicado por Elsevier España, S.L.U. Este es un artículo Open Access bajo la licencia CC BY-NC-ND (http://creativecommons.org/licenses/by-nc-nd/4.0/). 


\section{KEYWORDS}

Heart failure;

Functional status;

Validity;

Reliability;

Duke Activity Status

Index

\section{Validation of a questionnaire for Measuring functional capacity in patients with heart failure in Colombia}

\begin{abstract}
Objectives: To validate the Duke Activity Status Index questionnaire in patients with heart failure in Colombia.

Methods: A translation to Spanish and a cultural adaptation was performed on the Duke Activity Status Index (DASI) questionnaire. A total 135 patients were included to carry out the content validity, with a confirmatory factor analysis. The convergent and divergent construct validity, and the criteria validity was evaluated, comparing New York Heart Association (NYHA) classification groups, and the correlations with the "Minnesota Living with Heart Failure Questionnaire" quality of life questionnaire, the SF-36 quality of life generic scale, and a questionnaire on the evaluation of disability by the World Health Organisation (WHO-DAS II). Internal consistency and inter-observer reliability was evaluated, as well as the sensitivity to change at one, three, and six months.

Results: The scale is adjusted to two domains in the content validity. The convergent and divergent validity was verified, with significant correlations of the DASI with the scales that it was compared against. The criteria validity of the DASI with the NYHA classification showed some relationship, but it was not statistically significant. The Cronbach alpha was 0.69 in the internal consistency, and the inter-class correlation coefficients for inter-observer and within-observer reliability were 0.81 and 0.90 , respectively.

Conclusions: This version of the Duke Activity Status Index (DASI) in Spanish is valid and reliable for patients with heart failure in Colombia.

(c) 2018 Sociedad Colombiana de Cardiología y Cirugía Cardiovascular. Published by Elsevier España, S.L.U. This is an open access article under the CC BY-NC-ND license (http:// creativecommons.org/licenses/by-nc-nd/4.0/).
\end{abstract}

\section{Introducción}

En los pacientes con falla cardíaca hay disminución de la tolerancia al ejercicio, disnea y fatiga como primeras manifestaciones clínicas ${ }^{1}$; se afecta la capacidad funcional que describe la posibilidad de una persona para realizar las actividades diarias que requieren esfuerzo físico ${ }^{2}$. En Latinoamérica, la incidencia de falla cardiaca es de 199/ 100.000 personas-años; la prevalencia es del $1 \%$, la frecuencia de rehospitalización es de $33 \%$ a los tres meses, y la tasa de mortalidad al año de $24,5 \%^{3}$. En Colombia, la prevalencia en la población general es de $2,3 \%$. El aumento de la prevalencia incrementa los costos de su atención y conduce a deterioro funcional y de la calidad de vida ${ }^{4,5}$.

La prueba de esfuerzo cardiopulmonar (CPET) es la prueba estándar para la medición de la capacidad funcional en pacientes con falla cardiaca. Es útil en la clasificación y el seguimiento de pacientes y como predictor de pronóstico e indicación de pertinencia de trasplante cardíaco y soporte circulatorio ${ }^{6}$. Evalúa el consumo de oxígeno pico $\left(\mathrm{VO}_{2}\right.$ pico) que ha mostrado ser buen predictor de pronóstico de mortalidad ${ }^{1,6}$. Por tanto, la medición del $\mathrm{VO}_{2}$ pico y la capacidad funcional es parte fundamental de la evaluación integral de la salud cardiovascular. Sin embargo, la CPET tiene como desventajas los costos, el tiempo, la disponibilidad y los riesgos. Otras pruebas para medir la capacidad funcional incluyen la prueba de caminata de seis minutos que mide la distancia recorrida en seis minutos, y es útil para predecir capacidad física, hospitalizaciones y muerte en pacientes con falla cardiaca ${ }^{7}$, así como la prueba funcional con ejercicio submáximo, que determina la capacidad funcional en equivalentes metabólicos (MET) según el protocolo de ejercicio de Bruce o Bruce modificado ${ }^{8,9}$. La New York Heart Association (NYHA) hace una clasificación funcional de los pacientes con falla cardiaca en cuatro clases basadas en las limitaciones en las actividades de la vida diaria debidas a síntomas cardíacos, es fácil de usar y se ha visto relación significativa entre el $\mathrm{VO}_{2}$ pico y la $\mathrm{NYHA}^{10}$.

El índice de estado de actividad de Duke (DASI) es un cuestionario autoadministrado que mide la capacidad funcional de manera económica y simple; permite el seguimiento y es útil en la práctica clínica. Fue creado en 1989 en el Duke University Medical Center, en Carolina del Norte en idioma inglés ${ }^{11}$. En su versión original está conformado por 12 preguntas sobre aspectos de la función física relacionados con las actividades de la vida diaria. Permite hacer un cálculo de la capacidad funcional reportando resultados en $\mathrm{ml}$ de $\mathrm{O}_{2} / \mathrm{kg} /$ minuto con buena correlación con el $\mathrm{VO}_{2}$ pico, obtenido en la CPET y a su vez puede compararse con los $\mathrm{MET}^{11}$.

El DASI ha sido validado en enfermedad coronaria ${ }^{12}$, falla cardiaca $^{13}$, enfermedad pulmonar obstructiva crónica ${ }^{14}$ y enfermedad renal crónica ${ }^{15}$. Se ha encontrado que tiene buena sensibilidad al cambio por lo que es útil en la toma de decisiones clínicas ${ }^{16}$. En estudios de sus propiedades psicométricas en falla cardiaca se encontró una buena consistencia interna (alfa de Cronbach $=0,86$ ), en la validez de criterio se encontraron diferencias significativas entre los grupos de NYHA y en la validez de constructo se hallaron correlaciones significativas con la escala de calidad de 
vida asociada a la salud "Minnesota Living with Heart Failure Questionnaire', $(r=-0,64)$ y con síntomas depresivos evaluados con el "Beck Depression Inventory-II" $(r=-0,44)^{13}$.

El propósito de este estudio fue hacer la validación para Colombia de la versión original del Índice de estado de actividad de Duke - DASI, para aportar un instrumento para la evaluación, el seguimiento de pacientes con falla cardiaca y el desarrollo de investigaciones.

\section{Materiales y métodos}

Este fue un estudio observacional de validación de una escala, prospectivo en tres momentos: el primero en el que se hizo la traducción, una prueba piloto y la adaptación cultural; el segundo en el que se hizo la evaluación de la consistencia interna, la confiabilidad, la validez de criterio y la validez de constructo convergente y divergente; y el tercero longitudinal para la evaluación de la sensibilidad al cambio. Fue un estudio realizado de forma simultánea con la validación de la escala, "Minnesota living with heart failure questionnaire (MLHFQ) de calidad de vida en Colombia".

El estudio se llevó a cabo en la Clínica Las Américas de la ciudad de Medellín, Colombia. La recolección de la información se hizo desde octubre de 2013 hasta septiembre de 2016. Se siguieron los lineamientos de la declaración de ética médica de Helsinki, y según la resolución 8430 de 1993 de la República de Colombia se considera un estudio de riesgos mínimos. Fue aprobado por el comité de ética de la Clínica Las Américas y se llevó a cabo con la autorización del autor del DASI con quien se estableció comunicación por medio de correo electrónico. Los pacientes fueron informados del objetivo del estudio y se realizó un consentimiento informado.

Se incluyeron pacientes mayores de 16 años, que hablaran español, con diagnóstico de falla cardiaca sistólica o diastólica con una fracción de eyección $\leq 50 \%$ en la ecocardiografía, que asistieron a un programa de rehabilitación cardíaca. Se excluyeron aquellos con trastornos psiquiátricos y/o alteraciones cognitivas y/o la presencia de alguna de las siguientes comorbilidades: cáncer, enfermedad pulmonar obstructiva crónica o insuficiencia renal crónica. Los pacientes fueron captados en forma secuencial si cumplían con los criterios de ingreso.

Los datos fueron recolectados por una enfermera y una terapeuta física, cada una con más de diez años de entrenamiento en elaboración de entrevistas. Dos médicos fisiatras con experiencia en rehabilitación cardíaca, una terapeuta física especialista en rehabilitación cardíaca y una terapeuta ocupacional con experiencia en la atención de este tipo de pacientes realizaron la clasificación funcional de la New York Heart Association, las pruebas funcionales y el programa de rehabilitación cardíaca.

Se aplicaron los siguientes instrumentos:

1. Un formulario de recolección de la información que contenía datos de identificación, características sociodemográficas y clínicas.

2. El índice de estado de actividad de Duke (DASI).

3. La escala de calidad de vida "Minnesota Living with Heart Failure Questionnaire"' (MLHFQ).

4. La escala genérica de calidad de vida SF-36.
5. Un cuestionario de evaluación de la discapacidad de la Organización Mundial de la Salud (WHO-DAS II).

Todos los datos fueron tomados al inicio del estudio y luego se hizo seguimiento con el DASI y la prueba de ejercicio submáxima al mes, tres y seis meses. La calidad de los datos fue evaluada por una coinvestigadora y una profesional en sistemas de información.

El Índice de estado de actividad de Duke - DASI tiene un dominio y 12 ítems: cuidado personal (un ítem), deambulación (cuatro ítems), actividades del hogar (tres ítems), actividades de jardín (un ítem), relaciones sexuales (un ítem) y actividades recreativas (dos ítems); cada ítem del DASI tiene un peso ponderado según el consumo de energía de la actividad evaluada. Se tomaron cuatro opciones de respuesta: lo hizo sin dificultad, lo hizo pero con dificultad, no lo hizo por problemas de salud cardiovascular, no lo hizo por cualquier otra razón. La puntuación total final oscila entre 0 (peor estado funcional) y 58,2 (mejor estado funcional) ${ }^{11}$ (ver anexos 1 y 2). Luego se asigna a cada pregunta un puntaje según el anexo 3 si la respuesta es "si, sin dificultad" y no se asignan puntos en caso contrario. Se hace la sumatoria y se aplica la formula $\mathrm{VO}_{2}=0,43 * \mathrm{DASI}+9,6$, de acuerdo con lo propuesto por el autor de la versión original ${ }^{11}$.

El "Minnesota Living with Heart Failure Questionnaire (MLHFQ)"' evalúa la calidad de vida relacionada con la salud y consta de 21 ítems sobre el impacto de la falla cardiaca en diversos aspectos de la vida cotidiana (aspectos físicos, psicológicos y sociales). El rango total de puntuaciones posibles es 0 , mejor calidad de vida, a 105, peor calidad de vida ${ }^{17-19}$.

El SF36 es un instrumento genérico de calidad de vida validado en Colombia, y está compuesto por 36 preguntas que miden los siguientes dominios: función física (FF), desempeño físico (DF), dolor corporal (DC), salud general (SG), vitalidad (VT), función social (FS), desempeño emocional (DE) y salud mental (SM), además tiene un ítem adicional que pregunta sobre el cambio del estado de salud general en el último año. El resultado total va de 0-100 puntos; a mayor puntuación mejor es el estado de salud ${ }^{20-22}$.

El "World Health Organization - Disability Assessment Schedule (WHO-DAS)"' es un instrumento genérico de medida de la discapacidad compuesto por 36 ítems que evalúan las seis dimensiones esenciales del funcionamiento de un individuo según la Organización Mundial de la Salud. El puntaje final oscila entre 0 y 100 , donde 0 es estar sin discapacidad y 100 es la discapacidad completa ${ }^{23}$.

\section{Traducción y adaptación cultural}

Para este proceso se conformó un grupo constituido por dos traductores oficiales que hablan castellano como lengua nativa e inglés con buen dominio, quienes hicieron las traducciones independientes de la versión original del DASI. Se realizó una prueba piloto con 15 pacientes.

\section{Validez de apariencia}

Se tuvo en cuenta la opinión del grupo de investigación y los resultados de la prueba piloto. Se escogió la versión de 12 preguntas por considerar que evaluaba mejor la 
capacidad funcional y la función sexual. Se decidió adoptar la escala de respuestas de 4 opciones.

\section{Validez de contenido}

Se incluyeron 135 participantes que entraron a la investigación de validación del MLHFQ, suficientes para la validación de contenido del DASI que tiene 12 ítems (10 pacientes por ítem más $10 \%$ por posibles pérdidas).

Se evaluó la estructura propuesta por Xiuzhen Fan $^{13}$ por medio de un análisis factorial confirmatorio. Para la valoración del ajuste del modelo se utilizaron el "Root Mean Square Error of Approximation"' (RMSEA), que debe ser inferior a 0,07, el "Comparative fit index" (CFI) y el "Tucker-Lewis index" (TLI) que deben ser superiores a $0,90^{24,25}$. Este análisis se realizó con el software MPLUS versión $7.4^{26}$.

\section{Validez de criterio}

Se compararon los resultados del DASI entre los pacientes por clasificación funcional NYHA. El tamaño de muestra se calculó de acuerdo con los reportes de la literatura ${ }^{13}$, con el software tamaño de muestra 1.1 de la Pontificia Universidad Javeriana $^{27}$, según los siguientes datos: error tipo I de 0,05 y error tipo II de 0,2 , promedio de pacientes $\mathrm{NYHA} \mathrm{I} / \mathrm{II}=22$, promedio de los pacientes NYHA III/IV = 10, diferencia entre el promedio de los dos grupos 12; desviación estándar de 12,1 para una hipótesis de dos colas, obteniendo un total de 55 por grupo, más $22 \%$ de posibles pérdidas, para un total de 135 pacientes. Los grupos se compararon utilizando el ANOVA, con un nivel de significación un valor alfa $=0,05$.

\section{Validez de constructo}

La validez convergente se evaluó utilizando las correlaciones entre el DASI y la dimensión física del MLHFQ, los dominios de FF y DF del SF36 y la capacidad de moverse a su alrededor, las actividades laborales y la puntuación total del WHO-DAS. Las correlaciones convergentes miden conceptos similares y se espera que presenten el valor absoluto de los coeficientes de correlación de 0,4 o mayores. La validez divergente se hizo a partir de las correlaciones de DASI con las dimensiones de SM y DE del SF36, la dimensión emocional del MLHFQ y participación en sociedad, relaciones con otras personas y comprensión y comunicación del WHO-DAS ${ }^{28}$; en las correlaciones divergentes se espera que presenten un valor absoluto de los coeficientes de correlación menores de 0,4. El tamaño de muestra se calculó con el software de la Pontificia Universidad Javeriana ${ }^{27}$ con error tipo I: 0,05, error tipo II: 0,2; número de métodos: dos, coeficiente para la hipótesis nula: 0,4, coeficiente para la hipótesis alterna: 0,6 . Número de pacientes: 87 más el $35 \%$ por posibles pérdidas. El análisis estadístico se hizo mediante la matriz de correlaciones de Pearson entre el DASI y las dimensiones del SF-36 y del MLHFQ (multi-trait multi-method matrix).

\section{Consistencia interna y fiabilidad ínter-e intraobservador}

Se evaluó mediante el coeficiente Kuder Richardson fórmula 20 (KR-20) para ítems dicotómicos. Se aceptaron valores entre 0,7 y 0,9. Se aplicó a 135 participantes calculados para la validez de constructo que es el mayor tamaño de muestra. Para la fiabilidad interobservador, se aplicó la encuesta DASI al inicio y luego de 48 horas en un grupo de pacientes estables; la segunda aplicación se hizo por un profesional diferente e independiente del primer evaluador. El cálculo de tamaño de muestra se realizó con base en los siguientes parámetros: error tipo I: 0,05, error tipo II: 0,2. Coeficiente de correlación intraclase $(\mathrm{CCl})$ para la hipótesis nula de 0,6 y para la hipótesis alterna de 0,8 , hipótesis de dos colas más el $10 \%$ de posibles pérdidas, para un total de 44 pacientes. Para la fiabilidad intraobservador, se aplicó el DASI por el mismo entrevistador al inicio y luego de 14 a 16 días en un grupo de pacientes estables. El cálculo de tamaño de muestra se hizo teniendo en cuenta los siguientes parámetros: error tipo I 0,05 , error tipo II 0,2. CCl para la hipótesis nula de 0,6 y para la hipótesis alterna 0,8 , hipótesis de dos colas más el $10 \%$ de posibles pérdidas, para un total de 44 pacientes. Los análisis estadísticos se efectuaron con SPSS 20.0.

\section{Sensibilidad al cambio}

Se evaluó la capacidad del DASI para medir los cambios en la capacidad funcional después de un programa de rehabilitación cardíaca al mes, tres y seis meses. El tamaño de muestra se calculó con los siguientes parámetros ${ }^{11,27,29}$ : error tipo I: 0,05, desviación estándar: 13,49, distancia a la media poblacional: 3,98 , se obtuvo un tamaño de la muestra de 32 pacientes más un $20 \%$ por posibles pérdidas lo que equivale a 39 pacientes; se incluyeron 70 pacientes que fue la misma cantidad incluida en esta etapa en el estudio de validación del MLHFQ. El análisis estadístico se hizo por medio de la prueba de Wilcoxon. Se calculó el tamaño del efecto de Cohen; un tamaño del efecto de 0,8 es considerado alto, 0,5 moderado y 0,2 bajo $^{30}$. Adicionalmente, se calculó el mínimo cambio detectable (MCD), el cual se deriva del error estándar de la media (EEM) que se calcula con la siguiente fórmula. $E E M=D E_{\text {basal }} x \sqrt{1-R}$; donde $D E$ basal es la desviación estándar de la medición inicial y $\mathrm{R}$ el coeficiente de fiabilidad intraevaluador. El MCD se calcula de la siguiente manera: EEM $x$ z-score $x \sqrt{2}$. El z-score se asumió para un valor del $95 \%$ de confianza. Así, si un paciente presenta un valor superior al MCD se puede decir con un $95 \%$ de confianza que el cambio es importante desde el punto de vista clínico más allá del error de medida ${ }^{31,32}$.

\section{Resultados}

\section{Prueba piloto}

Se tomó una muestra de 15 pacientes con edad promedio de 60 años, 9 eran hombres (60\%). Con la aplicación de la prueba piloto se concluyó incluir un párrafo introductorio acorde con las opciones de respuesta, cambiar las respuestas de dicotómica a cuatro opciones (lo hizo sin dificultad, 


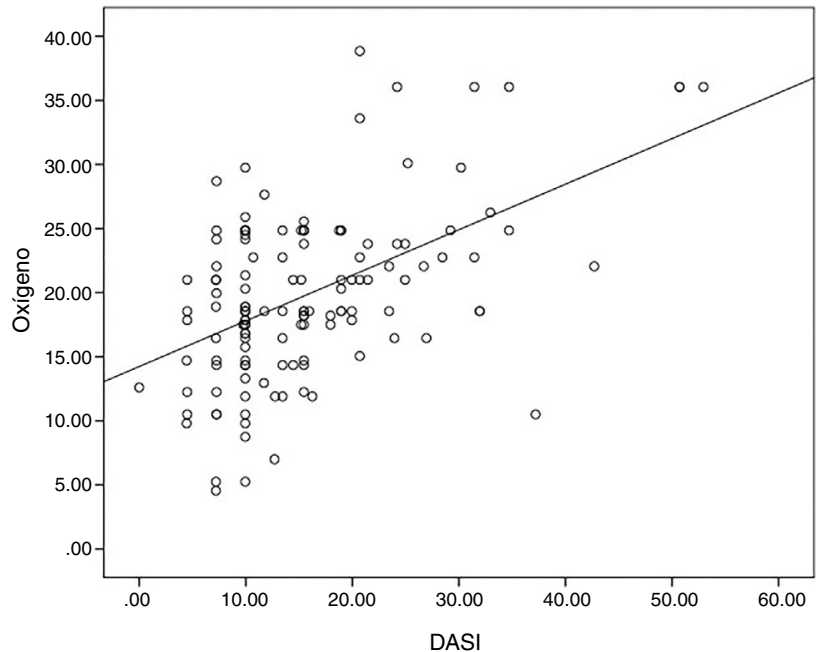

Figura 1 Gráfico de dispersión consumo máximo de oxígeno y puntaje DASI. A medida que aumenta el consumo de oxígeno incrementa el puntaje del DASI. El consumo de oxígeno se calculó con la fórmula $\mathrm{VO}_{2}=\left(0,355^{*} \mathrm{DASI}\right)+14,24$ (Coeficiente de determinación $\left.r^{2}=0,263\right)$.

lo hizo pero con dificultad, no lo hizo por problemas de salud cardiovascular, no lo hizo por cualquier otra razón) y en la pregunta cuatro poner "¿Puede subir unas escalas o una loma?' . Se decidió poner cuatro opciones de respuesta debido a las opiniones de los pacientes que participaron en la prueba piloto quienes consideraron que las opciones "si y no" no daban posibilidades más amplias de respuesta.

\section{Características sociodemográficas y clínicas}

El DASI se aplicó a 135 pacientes. La tabla 1 presenta las características demográficas y clínicas según la NYHA. La mayor proporción de personas incluidas fueron hombres $(66,7 \%)$, con edad promedio de 64,54 años; se tuvo una mayor cantidad de pacientes entre 55 y 74 años $(63,8 \%)$. Las puntuaciones totales del DASI tuvieron un promedio de $15,7 \pm 9,62$. La distribución según la clasificación funcional se observa en la tabla 1 . Respecto a la ocupación, el $62,2 \%$ se reintegraron, el $52,6 \%$ lo hicieron con modificaciones y el $9,6 \%$ sin modificaciones. La realización previa de actividad física también tuvo relación con la clasificación de la NYHA: mayor cantidad de pacientes de la NYHA I hacía deporte $(59,4 \%$ vs. $40,6 \%)$ mientras que en la clase funcional NYHA III la relación fue inversa ( $25 \%$ vs. $75 \%$ ). Tenían antecedentes de diabetes $(25,2 \%)$, obesidad $(18,5 \%)$ y dislipidemia $(77 \%)$.

La conversión del DASI a $\mathrm{VO}_{2}$ pico se hizo mediante una fórmula matemática basada en un modelo de regresión lineal según lo propuesto por los autores ${ }^{11}$. La figura 1 muestra la relación entre la $\mathrm{DASI}$ y el $\mathrm{VO}_{2}$ pico medido en la prueba de ejercicio submáxima al inicio, obteniéndose el modelo $\mathrm{VO}_{2}=\left(0,355^{*} \mathrm{DASI}\right)+14,24$ (coeficiente de determinación $r^{2}=0,263$ ). A medida que se aumenta el consumo de oxígeno, incrementa el DASI.

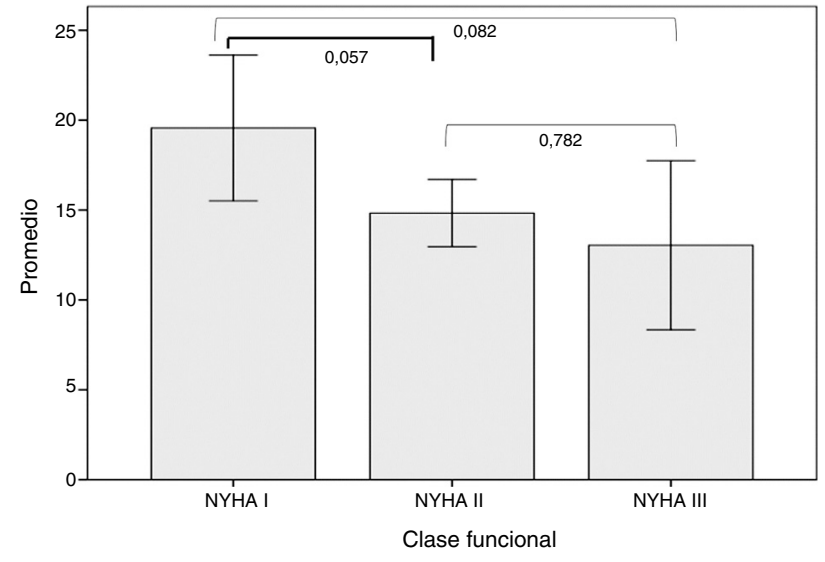

Figura 2 Promedios del DASI de acuerdo con la clase funcional y el valor de $\mathrm{p}$. Las barras representan el intervalo de confianza del $95 \%$.

\section{Validez de contenido}

Se realizó un análisis factorial confirmatorio con base en el trabajo de Xiuzhen Fan de dos dominios: uno de actividades pesadas y otro de actividades livianas (tabla 2$)^{13}$. El modelo mostró un ajuste adecuado con un valor de Chi cuadrado $=61,60 ; p=0,1469$, un RMSEA $=0,039, C F I=0,952$ y un $\mathrm{TLI}=0,938$. De acuerdo con los índices de modificación se agregaron las correlaciones entre los errores de los ítems 8 y 11 y 8 y 12 .

\section{Validez de criterio}

En la tabla 3 y la figura 2 se presentan los resultados del DASI según la NYHA. Se encontró que la media del DASI en los pacientes con clase funcional de la NYHA I, fue mayor a la de los NYHA II y III y en los NYHA II fue mayor que los de NYHA III, aunque la diferencia no fue significativa $(p>0,05)$.

\section{Validez de constructo}

La tabla 4 presenta los resultados de la evaluación de la validez de constructo tipo convergente y discriminante entre el DASI, y las dimensiones del SF-36, WHO-DAS y MLHFQ. Se encontraron coeficientes de correlación mayores a 0,40 y estadísticamente significativos $(p<0,001)$ entre el DASI y las dimensiones FF y DF del SF-36, con la dimensión física del MLHFQ y con los dominios del WHO-DAS que evalúan capacidad para moverse alrededor, actividades laborales y puntaje total. En la validez divergente se encontraron coeficientes de correlación menores a 0,4 entre el DASI y las dimensiones DE y SM del SF-36, compresión y comunicación del WHO-DAS y la dimensión emocional del MLHFQ.

\section{Consistencia interna, fiabilidad inter- e intra observador}

La escala global tuvo un coeficiente KR-20 de 0,72 IC 95\% $(0,64-0,79)$. En la fiabilidad interobservador fue de 0,81 IC $95 \%(0,67-0,90)$ y en la intraobservador de 0,90 IC $95 \%$ $(0,82-0,95)$. 
Tabla 1 Características demográficas y clínicas según clase funcional de la NYHA

\begin{tabular}{|c|c|c|c|c|}
\hline \multirow[t]{2}{*}{ Variable } & \multicolumn{3}{|c|}{ Clase funcional NYHA } & \multirow[t]{2}{*}{ Total } \\
\hline & $I(n=32)$ & II $(n=87)$ & III $(n=16)$ & \\
\hline \multicolumn{5}{|l|}{ Género* } \\
\hline Masculino & $21(65,6)$ & $61(70,1)$ & $8(50)$ & $90(66,7)$ \\
\hline \multicolumn{5}{|l|}{ Seguridad Social } \\
\hline Contributivo & $32(100)$ & $85(97,7)$ & $15(93,8)$ & $132(97,8)$ \\
\hline \multicolumn{5}{|l|}{ Entidad" } \\
\hline EPS & $28(87,5)$ & $81(93,1)$ & $15(93,8)$ & $124(91,9)$ \\
\hline \multicolumn{5}{|c|}{ Tiempo evolución en años del último evento* } \\
\hline$<0$ igual 1 & $26(81,3)$ & $73(83,9)$ & $14(87,5)$ & $113(83,7)$ \\
\hline$>1$ año & $5(15,6)$ & $13(14,9)$ & $2(12,5)$ & $20(14,8)$ \\
\hline Sin dato & $1(3,1)$ & $1(1,2)$ & $0(0)$ & $2(1,5)$ \\
\hline \multicolumn{5}{|l|}{ MET } \\
\hline $1-4,9$ & $5(15,6)$ & $27(31)$ & $9(56,3)$ & $41(30,4)$ \\
\hline $5-7$ & $16(50)$ & $39(44,8)$ & $6(37,5)$ & $61(45,2)$ \\
\hline$>7$ & $11(34,4)$ & $21(24,1)$ & $1(6,3)$ & $33(24,4)$ \\
\hline \multicolumn{5}{|l|}{ Diagnóstico* } \\
\hline Enfermedad coronaria & $25(78,1)$ & $60(69)$ & $10(62,5)$ & $95(70,4)$ \\
\hline Valvular & $1(3,1)$ & $7(8,0)$ & $2(12,5)$ & $10(7,4)$ \\
\hline Otros & $6(18,8)$ & $20(23)$ & $4(25,0)$ & $30(22,2)$ \\
\hline \multicolumn{5}{|l|}{ Fracción de eyección" } \\
\hline $40-50$ & $16(50,0)$ & $25(28,7)$ & $3(18,8)$ & $44(32,6)$ \\
\hline$<40$ & $16(50,0)$ & $62(71,3)$ & $13(81,3)$ & $91(67,4)$ \\
\hline \multicolumn{5}{|l|}{ Reintegro laboral } \\
\hline Con modificaciones & $17(53,1)$ & $46(52,9)$ & $7(43,8)$ & $70(51,9)$ \\
\hline Sin modificaciones & $4(12,5)$ & $7(8,0)$ & $2(12,5)$ & $13(9,6)$ \\
\hline Reubicación laboral & $0(0,0)$ & $1(1,1)$ & $0(0,0)$ & $1(0,7)$ \\
\hline DASI $/ 58,2^{* *}$ & $19,6 \pm 11,2$ & $14,8 \pm 8,8$ & $13 \pm 8,82$ & $15,7 \pm 9,62$ \\
\hline \multicolumn{5}{|l|}{$M L H F Q^{* * *}$} \\
\hline Dimensión física (0-40) & $7,9 \pm 7,4$ & $9,8 \pm 8,0$ & $12,6 \pm 8,2$ & $9,7 \pm 7,9$ \\
\hline \multicolumn{5}{|l|}{$S F-36^{* *}$} \\
\hline Función física (0-100) & $70,3 \pm 18,4$ & $57,8 \pm 21,4$ & $57,2 \pm 24,8$ & $60,7 \pm 21,7$ \\
\hline Desempeño físico (0-100) & $50,6 \pm 30,8$ & $39,5 \pm 30,0$ & $41,5 \pm 38,6$ & $42,3 \pm 31,4$ \\
\hline WHO-DAS $(0-100)^{* * *}$ & $18,9 \pm 17,7$ & $12,6 \pm 12,4$ & $14,7 \pm 12,8$ & $14,7 \pm 13,9$ \\
\hline
\end{tabular}

\section{Sensibilidad al cambio}

Los tamaños del efecto fueron estadísticamente significativos $(p<0,001)$ con valores al mes, al tercer mes y al sexto de $0,45,1,01$ y 0,92 . El MCD significativo fue de 8,4 puntos.

\section{Discusión}

Este estudio encontró un adecuado rendimiento del DASI para medir la capacidad funcional en pacientes colombianos con falla cardiaca, la validez convergente y divergente fue establecida por la confirmación de las hipótesis planteadas con el MLFHQ, SF-36 y WHO-DAS, se encontró una adecuada fiabilidad y consistencia interna.

En la estructura propuesta por Xiuzhen $\mathrm{Fan}^{13}$ del DASI se encontraron dos dimensiones, una de ellas relacionada con actividades que implican menor consumo de energía y la otra con actividades con mayor consumo de energía. En este estudio se confirmó esto, observando en las preguntas del dominio de actividades livianas un gran porcentaje de pacientes que respondió afirmativamente, en el otro dominio ocurrió lo contrario con pocos pacientes que podían llevar a cabo ítems como correr distancias cortas 
Tabla 2 Análisis de contenido. Factores derivados del análisis factorial confirmatorio

\begin{tabular}{lll}
\hline Ítem & Factor 1: actividades pesadas & Factor 2: actividades livianas \\
\hline Ítem 4. Subir escalas o loma & 0,33 & \\
Ítem 5. Correr distancias cortas & 0,66 & \\
Ítem 8. Labores domésticas pesadas & 0,53 & \\
Ítem 9. Labores de jardín & 0,58 & 0,37 \\
Ítem 10. Relaciones sexuales & 0,79 & 0,32 \\
Ítem 11. Actividades recreativas & 0,29 & 0,18 \\
Ítem 12. Deportes fatigantes & & 0,30 \\
Ítem 1. Autocuidado & & 0,35 \\
Ítem 2. Caminar en casa & & 0,72 \\
Ítem 3. Caminar 1 o 2 cuadras & & \\
Ítem 6. Labores domésticas sencillas & & \\
Ítem 7. Labores domésticas complejas & & \\
\hline
\end{tabular}

Tabla 3 Comparación de los resultados del DASI según clase funcional NYHA I, II, II

\begin{tabular}{llll}
\hline & Media $(\mathrm{DE})$ & Diferencia de medias Error estándar & Valor de $\mathrm{p}$ \\
\hline NYHA I vs NYHA II & $19,57(11,24)$ vs $14,84(8,80)$ & $4,73(1,95)$ & 0,057 \\
NYHA II vs NYHA III & $14,84(8,80)$ vs $13,04(8,82)$ & $1,80(2,57)$ & 0,782 \\
NYHA I vs NYHA III & $19,57(11,24)$ vs $13,04(8,82)$ & $6,53(2,89)$ & 0,082 \\
\hline
\end{tabular}

NYHA, New York Heart Association; DE: desviación estándar

Tabla 4 Validez de constructo tipo convergente y discriminante entre el DASI, y las dimensiones del SF-36, WHO-DAS y el MLHFQ

\begin{tabular}{|c|c|c|c|}
\hline & \multirow[t]{2}{*}{ Dominios } & \multicolumn{2}{|l|}{ DASI ${ }^{\S}$} \\
\hline & & Coeficiente de correlación & Valor $\mathrm{p}$ \\
\hline \multirow{9}{*}{ SF-36 } & Función física & $0,73^{*}$ & $<0,001$ \\
\hline & Desempeño físico & $0,50^{*}$ & $<0,001$ \\
\hline & Desempeño emocional & $0,30^{*}$ & $<0,001$ \\
\hline & Salud mental & $0,33^{*}$ & \\
\hline & Cambio de salud & 0,12 & 0,17 \\
\hline & Dolor corporal & $0,34^{*}$ & 0,00 \\
\hline & Función social & $0,40^{*}$ & 0,00 \\
\hline & Salud general & $0,44^{*}$ & 0,00 \\
\hline & Vitalidad & $0,49^{*}$ & 0,00 \\
\hline \multirow[t]{8}{*}{ WHO-DAS $§ \S \S$} & Comprensión y comunicación & $-0,38^{*}$ & 0,00 \\
\hline & Capacidad para moverse a su alrededor & $-0,55^{*}$ & 0,00 \\
\hline & Cuidado personal & $-0,43^{*}$ & 0,00 \\
\hline & Relaciones con otras personas & $-0,44^{*}$ & 0,00 \\
\hline & Actividades de la vida diaria & $-0,48^{*}$ & 0,00 \\
\hline & Actividades laborales & $-0,61^{*}$ & 0,00 \\
\hline & Participación en sociedad & $-0,44^{*}$ & 0,00 \\
\hline & Puntuación global WHO-DAS & $-0,66^{*}$ & 0,00 \\
\hline \multirow[t]{3}{*}{ MLHFQ $§ \S \S$} & Dimensión física & $-0,60^{*}$ & 0,00 \\
\hline & Dimensión emocional & $-0,12$ & 0,18 \\
\hline & Puntaje total Minnesota & $-0,65^{*}$ & 0,00 \\
\hline
\end{tabular}

${ }^{\S}$ DASI, Índice de estado de actividad de Duke.

$\S \S$ SF-36, cuestionario de calidad de vida relacionada con la salud MLHFQ.

$\S \S$ WHO-DAS, World Health Organization Disability Assessment Schedule.

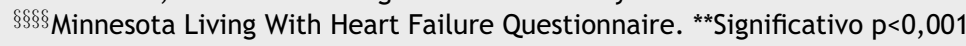


$(8,1 \%)$, labores domésticas pesadas $(4,4 \%)$ y deportes fatigantes $(0,7 \%)$; esta estructura de dos dominios es idéntica a la encontrada en el estudio en falla cardiaca de Xiuzhen Fan et al. y muy similar al estudio hecho en enfermedades cardíacas de Coutinho-Myrrha et al. ${ }^{33}$. Estos hallazgos sugieren que el DASI tiene dos dominios en pacientes con falla cardiaca.

La validez de criterio del DASI fue apoyada por mejor capacidad funcional en los pacientes de los grupos NYHA I vs. los NYHA II y III, sin embargo, no fueron estadísticamente significativos; en estudios previos de otras poblaciones de pacientes con falla cardiaca se ha confirmado esta correlación ${ }^{13,34}$.

Es probable que nuestros resultados se deban a que se contó con mayor población con clase funcional NYHA II y no se incluyeron pacientes con NYHA IV. Esto refleja las características de la población que con mayor frecuencia es remitida a rehabilitación cardíaca.

En la validez de constructo del DASI se encontraron buenas correlaciones convergentes y divergentes con el MLHFQ, SF-36 y WHO-DAS. Se han realizado estudios previos que han comparado el DASI y el puntaje total del MLHFQ encontrando correlaciones de $-0,64^{13}$ y $-0,55^{35}$ que son similares a los resultados hallados en este estudio y que indican que a mejor estado funcional, mejor calidad de vida asociada a la salud. Este estudio, muestra las correlaciones específicas con los dominios de los otros instrumentos seleccionados incluyendo el WHO-DAS II que es una escala basada en la Clasificación Internacional de Funcionamiento ${ }^{23}$. Este es el primer estudio que evalúa la validez convergente de estas dos escalas.

El DASI tuvo buena sensibilidad al cambio, con una magnitud del efecto moderada al mes, y grande a los tres y seis meses. Lo anterior refleja la respuesta fisiológica de reacondicionamiento de estos pacientes al alcanzar su máxima capacidad debido al entrenamiento; a los seis meses la respuesta se estabiliza lo que puede reflejar la buena adherencia a la actividad física. El tamaño del efecto ha sido medido en otros estudios con valores de 0,75 en pacientes con angioplastia y de 0,22 en pacientes estables ${ }^{12}$. El MCD significativo fue de 8,4 puntos, el cual es un resultado importante para tener en cuenta en el cambio que se espera en un estudio de intervención.

Le evaluación de la fiabilidad interevaluador e intraevaluador del DASI fue muy buena, $\mathrm{CCl} 0,81$ y 0,90 respectivamente y buena consistencia interna (alfa de Cronbach: 0,69). En otros estudios previos se ha informado la consistencia interna del DASI con coeficientes de alfa de Cronbach de 0,82 a 0,92 $13,33-36$, similares a los obtenidos en este estudio derivados de grupos de pacientes en estadios más graves de la enfermedad y con peor clase funcional.

Una de las limitaciones de este estudio es que la selección de pacientes, se hizo de manera consecutiva y no estratificada por lo cual no hubo pacientes con NYHA IV y el $64,4 \%$ de la muestra fue NYHA II. No se realizó la CPET, pero como fortaleza del estudio se hizo la prueba de esfuerzo submáxima que es una medida objetiva válida del estado funcional.

\section{Conclusión}

Esta es una escala funcional que se correlaciona en forma adecuada con la capacidad funcional; es de fácil aplicación en los diferentes niveles de atención. Puede ser útil para programas de falla cardiaca que se realicen en niveles de baja o mediana complejidad.

Para investigaciones futuras se debe evaluar la forma de calificación de cada ítem y como estas diferentes opciones se pueden calificar al menos en tres categorías: lo hizo, sin dificultad, lo hizo pero con dificultad, no lo hizo. Otras investigaciones necesarias en esta área serían cómo calificar y validar el $\mathrm{VO}_{2}$ en las diferentes actividades de la vida diaria y laboral para dar indicaciones más precisas a los pacientes.

\section{Financiación}

Esta investigación fue financiada con la estrategia de sostenibilidad 2016, 2017 de la Universidad de Antioquia y con recursos en especie de la Clínica Las Américas.

\section{Conflicto de intereses}

Ninguno.

\section{Agradecimientos}

A la Clínica Las Américas y a los profesionales del área de rehabilitación cardíaca por su disposición para participar en esta investigación.

A la enfermera Rocío Romero Llanos y a la fisioterapeuta Deysi Espinoza García.

\section{Anexo. Material adicional}

Se puede consultar material adicional a este artículo en su versión electrónica disponible en doi:10.1016/ j.rccar.2018.04.004.

\section{Bibliografía}

1. Arena R, Myers J, Williams MA, Gulati M, Kligfield P, Balady GJ, et al. Assessment of Functional Capacity in Clinical and Research Settings: A Scientific Statement From the American Heart Association Committee on Exercise, Rehabilitation, and Prevention of the Council on Clinical Cardiology and the Council on Cardiovascular Nursing. Circulation. 2007;116:329-43. Disponible en: http: / / circ.ahajournals.org/cgi/doi/10.1161/CIRCULATIONAHA. 106. 184461

2. Arena R, Cahalin LP, Borghi-Silva A, Phillips SA. Improving functional capacity in heart failure. Curr Opin Cardiol. 2014;29:467-74. Disponible en: http://content.wkhealth.com/ linkback / openurl?sid=WKPTLP:landingpage\&an $=00001573$ 201409000-00011

3. Ciapponi A, Alcaraz A, Calderón M, Matta MG, Chaparro M, Soto $\mathrm{N}$, et al. Carga de enfermedad de la insuficiencia cardiaca en América Latina: revisión sistemática y metanálisis. 
Rev Esp Cardiol. 2016;69:1051-60. Disponible en: http:// linkinghub.elsevier.com/retrieve/pii/S0300893216302469

4. Roger VL. Epidemiology of Heart Failure. Circ Res. 2013;113:646-59. Disponible en: http://circres.ahajournals. org/lookup/doi/10.1161/CIRCRESAHA.113.300268

5. Jaramillo C, Gómez E, Hernández E. Consenso Colombiano para el diagnóstico y tratamiento de la insuficiencia cardíaca crónica. Bogotá: Sociedad Colombiana de Cardiología y Cirugía Cardiovascular; 2014.

6. Balady GJ, Arena R, Sietsema K, Myers J, Coke L, Fletcher GF, et al. Clinician's Guide to cardiopulmonary exercise testing in adults: a scientific statement from the American Heart Association. Circulation. 2010;122:191-225. Disponible en: http:// circ.ahajournals.org/cgi/doi/10.1161/CIR.0b013e3181e52e69

7. Senior JM, Lugo LH, Díaz JS. Guía de Práctica Clínica (GPC) para la prevención, diagnóstico, tratamiento y rehabilitación de la falla cardíaca en población mayor de 18 años clasificación B, C y D [Internet]. 2016. Disponible en: http://gpc. minsalud.gov.co/gpc_sites/Repositorio/Conv_637/GPC_falla cardiaca/gpc_falla_cardiaca.aspx

8. Malhotra R, Bakken K, D’Elia E, Lewis GD. Cardiopulmonary exercise testing in heart failure. JACC: Heart Fail. 2016;4:607-16. Disponible en: http://linkinghub.elsevier. com/retrieve/pii/S2213177916301299

9. Kaminsky LA, Tuttle MS. Functional assessment of heart failure patients. Heart Fail Clin. 2015;11:29-36.

10. Rostagno C, Galanti G, Comeglio M, Boddi V, Olivo G, Gastone Neri Serneri G. Comparison of different methods of functional evaluation in patients with chronic heart failure. Eur J Heart fail. 2000;2:273-80. Disponible en: http://www.ncbi.nlm.nih.gov/pubmed/10938488

11. Hlatky MA, Boineau RE, Higginbotham MB, Lee KL, Mark DB, Califf RM, et al. A brief self-administered questionnaire to determine functional capacity (the Duke Activity Status Index). Am J Cardiol. 1989;64:651-4. Disponible en: http://www.ncbi.nlm.nih.gov/pubmed/2782256

12. Alonso J, Permanyer-Miralda G, Cascant P, Brotons C, Prieto $\mathrm{L}$, Soler-Soler J. Measuring functional status of chronic coronary patients. Reliability, validity and responsiveness to clinical change of the reduced version of the Duke Activity Status Index (DASI). Eur Heart J. 1997;18:414-9. Disponible en: http://www.ncbi.nlm.nih.gov/pubmed/9076377

13. Fan X, Lee KS, Frazier SK, Lennie TA, Moser DK. Psychometric testing of the Duke Activity Status Index in patients with heart failure. Eur J Cardiovasc Nurs. 2015;14:214-21. Disponible en: http://journals.sagepub.com/doi/10.1177/1474515114523354

14. Tavares L dos A, Barreto Neto J, Jardim JR, Souza GM da Ce, Hlatky MA, Nascimento OA. Cross-cultural adaptation and assessment of reproducibility of the Duke Activity Status Index for COPD patients in Brazil. J Bras Pneumol. 2012;38:684-91. Disponible en: http://www. ncbi.nlm.nih.gov/pubmed/23288112

15. Ravani P, Kilb B, Bedi H, Groeneveld S, Yilmaz S, Mustata S. The Duke Activity Status Index in Patients with Chronic Kidney Disease: A Reliability Study. Clin J Am Soc Nephrol. 2012;7:573-80. Disponible en: http://cjasn. asnjournals.org/cgi/doi/10.2215/CJN.07990811

16. Koch CG, Khandwala F, Cywinski JB, Ishwaran H, Estafanous FG, Loop FD, et al. Health-related quality of life after coronary artery bypass grafting: A gender analysis using the Duke Activity Status Index. J Thorac Cardiovasc Surg. 2004;128:284-95. Disponible en: http://linkinghub. elsevier.com/retrieve/pii/S0022522304001199

17. Middel B, Bouma J, de Jongste $M$, van Sonderen $E$, Niemeijer MG, Crijns $\mathrm{H}$, et al. Psychometric properties of the Minnesota Living with Heart Failure Questionnaire (MLHF-Q). Clin Rehabil. 2001;15:489-500. Disponible en: http://www.ncbi.nlm.nih.gov/pubmed/11594639
18. Heo S, Moser DK, Riegel B, Hall LA, Christman N. Testing the psychometric properties of the Minnesota Living with Heart Failure questionnaire. Nurs Res. 2005;54:265-72. Disponible en: http://www.ncbi.nlm.nih.gov/pubmed/16027569

19. Garin O, Soriano N, Ribera A, Ferrer M, Pont A, Alonso $\mathrm{J}$, et al. Validation of the Spanish version of the Minnesota Living with Heart Failure Questionnaire. Rev Esp Cardiol. 2008;61:251-9. Disponible en: http://www.ncbi.nlm. nih.gov/pubmed/18361898

20. Vilagut G, Ferrer M, Rajmil L, Rebollo P, Permanyer-Miralda $G$, Quintana JM, et al. The Spanish version of the Short Form 36 Health Survey: a decade of experience and new developments. Gac Sanit. 2004;19:135-50. Disponible en: http://www.ncbi.nlm.nih.gov/pubmed/15860162

21. Lugo LH, García HI, Gómez. C. Confiabilidad del cuestionario de calidad de vida en salud SF-36 en Medellín. Colombia. Rev Fac Nac Sal Pub. 2006;24:37-50.

22. García HI, Vera CY, Lugo LH. Calidad de vida relacionada con la salud en Medellín y su área metropolitana, con aplicación del SF-36. Rev Fac Nac Salud Pública. 2014;32:26-39. Disponible en: http://www.redalyc.org/pdf/120/12029652004.pdf

23. Vázquez BJ, Herrera CS, Vázquez BE, Gaite P. Cuestionario para la evaluación de discapacidad de la Organización Mundial de la Salud (Versión española del World Health Organization Disability Assessment Schedule II). Ministerio de Trabajo y Asuntos Sociales, Subdirección General de Información Administrativa y Publicaciones, Madrid; 2006.

24. Streiner DL, Norman, Geoffrey R, Cairney J. Health Measurement Scales. 5 a. Ed. Oxford Scholar; 2015.

25. Hu L, Bentler PM. Cutoff criteria for fit indexes in covariance structure analysis: Conventional criteria versus new alternatives. Struct Equ Model A Multidiscip J. 1999;6:1-55. Disponible en: http://www.tandfonline.com/ doi/abs/10.1080/10705519909540118

26. Muthén LK, Muthén BO. Mplus User's Guide. Eighth Edi. Los Angeles: Muthén \& Muthén; 2017.

27. Pérez A, Rodríguez MN, Gil JF RG. Tamaño de la muestra versión 1.1. Pontificia Universidad Javeriana. Unidad de Epidemiología Clínica. Disponible en: http://hermes. javeriana.edu.co/tamamu

28. Gottlieb SS, Kop WJ, Ellis SJ, Binkley P, Howlett J, O'Connor C, et al. Relation of Depression to Severity of Illness in Heart Failure (from Heart Failure And a Controlled Trial Investigating Outcomes of Exercise Training [HF-ACTION]). Am J Cardiol. 2009;103:1285-9. Disponible en: http:// linkinghub.elsevier.com/retrieve/pii/S0002914909001192

29. Nedeljkovic U, Krstic N, Varagic-Markovic S, Putnik S. Quality of life and functional capacity one year after coronary artery bypass graft surgery. Acta Chir lugosl. 2011;58:81-6. Disponible en: http://www.doiserbia.nb.rs/ Article. aspx?ID=0354-950X1103081N

30. Fritz CO, Morris PE, Richler JJ. Effect size estimates: Current use, calculations, and interpretation. J Exp Psychol Gen. 2012;141:2-18. Disponible en: http://doi.apa.org/ getdoi.cfm?doi=10.1037/a0024338

31. Beckerman H, Roebroeck ME, Lankhorst GJ, Becher JG, Bezemer PD, Verbeek AL. Smallest real difference, a link between reproducibility and responsiveness. Qual Life Res. 2001;10: 571-8.

32. D Vet HCW, Bouter LM, Bezemer PD, Beurskens AJ. Reproducibility and responsiveness of evaluative outcome measures. Theoretical considerations illustrated by an empirical example. Int J Technol Assess Health Care. 2001;17:479-87.

33. Coutinho-Myrrha MA, Dias RC, Fernandes AA, Araújo CG, Hlatky MA, Pereira DG, et al. Duke Activity Status Index for Cardiovascular Diseases: Validation of the Portuguese Translation. Arq Bras Cardiol. 2014;102:383-90. Disponible en: http://www.gnresearch.org/doi/10.5935/abc.20140031 
34. Parissis JT, Nikolaou M, Birmpa D, Farmakis D, Paraskevaidis I, Bistola V, et al. Clinical and Prognostic Value of Duke's Activity Status Index Along With Plasma BType Natriuretic Peptide Levels in Chronic Heart Failure Secondary to Ischemic or Idiopathic Dilated Cardiomyopathy. Am J Cardiol. 2009;103:73-5. Disponible en: http:// linkinghub.elsevier.com/retrieve/pii/S0002914908014513

35. Wu J-R, Lennie TA, Frazier SK, Moser DK. HealthRelated Quality of Life, Functional Status, and Cardiac
Event-Free Survival in Patients With Heart Failure. J Cardiovasc Nurs. 2016;31:236-44. Disponible en: http:// content. wkhealth.com/linkback/openurl?sid=WKPTLP: landingpage\&an $=00005082-201605000-00008$

36. Heo S, Moser DK, Lennie TA, Riegel B, Chung ML. Gender differences in and factors related to self-care behaviors: A cross-sectional, correlational study of patients with heart failure. Int J Nurs Stud. 2008;45:1807-15. Disponible en: http:// linkinghub.elsevier.com/retrieve/pii/S002074890800120X 